\title{
Mediastinal lymph node enlargement in idiopathic pulmonary fibrosis: relationships with disease progression and pulmonary function trends
}

Giacomo Sgalla ${ }^{1 *}$ (D), Anna Rita Larici ${ }^{2,3}$, Nicoletta Golfi ${ }^{1}$, Mariarosaria Calvello ${ }^{1}$, Alessandra Farchione ${ }^{2}$, Annemilia Del Ciello ${ }^{2}$, Francesco Varone ${ }^{1}$, Bruno lovene ${ }^{1}$, Riccardo Manfredi ${ }^{2,3}$ and Luca Richeldi ${ }^{1,4}$

\begin{abstract}
Background and objectives: Evidence of mediastinal Lymph Node Enlargement (LNE) on CT scan is a common finding in idiopathic pulmonary fibrosis (IPF). We sought to investigate whether the involvement of mediastinal lymph nodes is associated with accelerated disease progression, and explored the changes occurring in mediastinal lymph nodes during the radiological follow up of these patients.

Methods: This retrospective study included IPF patients referred to a single ILD centre in Italy. A consensus-based assessment of mediastinal LNE on chest CT scan was performed by two thoracic radiologists. Kaplan-Meier curves and multivariate Cox proportional hazards regression were used to assess hazard ratios for mortality and disease progression (defined as categorical FVC decline $\geq 10 \%$ ). The annualized rates of change in functional parameters for each patient were calculated using mixed linear models.

Results: The study population consisted of 152 IPF patients, of whom 135 (89\%) received antifibrotic treatment for IPF during the study follow up. Patients having evidence of 3 or more enlarged mediastinal lymph nodes on baseline CT scan showed increased rates of mortality (HR 5.03, 95\% Cl 1.86-13.62, $p \leq 0.001$ ) and significant disease progression ( $\mathrm{HR} 2.99,95 \% \mathrm{Cl} 1.22-7.33, p=0.17$ ) as compared to patients without LNE, after adjusting for GAP stage. Among 62 patients with LNE who underwent a follow up CT scan of the chest and received antifibrotic treatment, 57 (92\%) maintained evidence mediastinal LNE over time.
\end{abstract}

Conclusions: Diffuse mediastinal lymph node involvement predicts clinically meaningful functional deterioration in patients with IPF.

Keywords: Idiopathic pulmonary fibrosis, Interstitial lung disease, Lymphadenopathy

\footnotetext{
* Correspondence: giacomo.sgalla@gmail.com

'Dipartimento Scienze Gastroenterologiche, Endocrino-Metaboliche e

Nefro-Urologiche, Unità Operativa Complessa di Pneumologia, Fondazione

Policlinico Universitario "A. Gemelli" IRCCS, Rome, Italy

Full list of author information is available at the end of the article
}

(c) The Author(s). 2020 Open Access This article is licensed under a Creative Commons Attribution 4.0 International License, which permits use, sharing, adaptation, distribution and reproduction in any medium or format, as long as you give appropriate credit to the original author(s) and the source, provide a link to the Creative Commons licence, and indicate if changes were made. The images or other third party material in this article are included in the article's Creative Commons licence, unless indicated otherwise in a credit line to the material. If material is not included in the article's Creative Commons licence and your intended use is not permitted by statutory regulation or exceeds the permitted use, you will need to obtain permission directly from the copyright holder. To view a copy of this licence, visit http://creativecommons.org/licenses/by/4.0/. The Creative Commons Public Domain Dedication waiver (http://creativecommons.org/publicdomain/zero/1.0/) applies to the data made available in this article, unless otherwise stated in a credit line to the data. 


\section{Background}

The prognosis of Idiopathic pulmonary fibrosis (IPF), the most fatal among idiopathic interstitial pneumonias $[1,2]$, is generally poor with an estimated survival between 3 and 5 years from the time of diagnosis [3]. Despite two antifibrotic drugs, nintedanib and pirfenidone, proved to slow down the functional decline in IPF $[4,5]$, most patients still deteriorate despite treatment. As such, accurate monitoring of disease progression in IPF is crucial for predicting prognosis and optimizing management, including the appropriate timing of supportive care and the prompt referral for lung transplantation. Clinical findings, pulmonary function measures and computed tomography findings have been extensively studied to predict survival in IPF, either individually or by using composite scoring indices [6-9], however prognostication remains challenging in the individual patient due to the heterogeneity of IPF and the significant intrapatient variability of the disease behaviour [10]. Measurement of forced vital capacity (FVC) via spirometry has been accepted as the most feasible and reliable tool to monitor disease progression in these patients in clinical practice as well as in randomized controlled trials $[11,12]$, however it has not been proved of great utility in predicting future loss of pulmonary function [13, 14]. Visual evaluation and computer-based quantification of high-resolution computed tomography (HRCT) lung parenchymal patterns of fibrosis have been demonstrated to be strong predictors of outcome, particularly in IPF patients $[7,9,15-17]$, however their use in clinical practice is still limited. The evidence of mediastinal lymphadenopathies on chest CT scans inpatients with interstitial lung disease (ILD) have drawn the attention of researchers over the years, due to the putative role played by inflammation and immunity processes in pulmonary fibrosis. The frequency of this finding has been reported in up to $70 \%$ of patients with a usual interstitial pneumonia pattern $[18,19]$, and in up to $58 \%$ of IPF patients [20, 21]. Although the biology underpinning mediastinal lymph node enlargement in these patients has never been clarified, the presence of LNE on chest CT scans has been associated with increased all-cause mortality in IPF and other ILD [22, 23], suggesting a role as useful prognostic factor in fibrotic lung disease. An ongoing randomized clinical trial is currently exploring the efficacy of a human monoclonal antibody directed against the Bcell activating factor (BAFF) receptor on B cells in IPF patients (clinicaltrials.gov identifier NCT03287414). The study recognizes the presence of hilar/mediastinal lymphadenopathies on HRCT as one of the key inclusion criteria, based on the hypothesis that lymph node involvement may identify IPF patients with pronounced immune activation and who are therefore more likely to respond to the investigational treatment.
To date however, it is unknown whether the enlargement of mediastinal lymph nodes may be used to identify patients with more rapid functional deterioration, or to indicate response to currently available antifibrotic treatments.

In this study, we retrospectively evaluated the impact of LNE on mortality and disease progression in a cohort of IPF patients, and explored the changes occurring in mediastinal lymph nodes during the radiological follow up.

\section{Methods}

\section{Study population and groups}

All patients referred to the outpatient ILD clinics at Fondazione Policlinico Universitario "A. Gemelli" IRCCS in Rome between January 2014 and November 2018 were retrospectively screened for inclusion in the study. Patients were included if they received a multidisciplinary diagnosis of IPF as per diagnostic guidelines $[1,3]$ and if they had performed a chest CT scan within 1 year from diagnosis. Patients were excluded if they had either concurrent pulmonary infection at the time of the CT scan, acquired immunodeficiency syndrome (AIDS), unstable chronic heart disease or history of malignancy with less than 5 years of negative follow up. Subjects with no available Pulmonary Functional Tests (PFTs) were also excluded. The study was approved by the local ethics committee of Fondazione Policlinico Universitario "A. Gemelli" IRCCS in Rome.

The presence (LNE+) or absence (LNE-) of at least one mediastinal lymph node with short-axis diameter $\geq 10 \mathrm{~mm}$ on chest CT scan at baseline, as reviewed by thoracic radiologists, was used to define the groups for this study.

\section{Collection of study data}

The medical records at the ILD outpatient clinic Fondazione Policlinico Universitario "A. Gemelli" IRCCS in Rome were used to obtain clinical data up to April 2019. Demographics and medical history including smoking status, supplemental oxygen therapy, antifibrotic treatment and comorbidities were collected together with baseline and follow up lung function data such as absolute and percent predicted values of FVC and diffusion capacity for carbon monoxide $\left(\mathrm{DL}_{\mathrm{CO}}\right)$ were obtained. The Gender/Age/Physiology (GAP) score, that integrates patient-specific variables (sex, age, FVC, DLCO) [8], was calculated for each patient. The follow up time was calculated for each patient as the time interval between the first and the last functional test performed at the referral centre.

\section{Chest CT evaluation}

The CT exams evaluated for this study were performed at different centres, with some variability in 
reconstruction parameters used and contrast medium administration. Since most of CT studies were performed for the analysis of the lung parenchyma, CT scans without contrast medium administration were the majority and were preferred for the analysis when available. Images with a maximum slice thickness of $5 \mathrm{~mm}$ (range: $1-5 \mathrm{~mm}$ ), smooth reconstruction algorithm and soft tissue window setting (level 50/width $350 \mathrm{HU}$ ) were used for lymph node assessment. Mediastinal lymph node stations based on the International Association for the Study of Lung Cancer nomenclature were systematically assessed for enlarged lymph nodes [24]. Mediastinal lymph node measurements were provided using electronic calipers. Lymph nodes with a short-axis diameter $\geq 10 \mathrm{~mm}$ were reported as enlarged, as previously described in papers focused on ILD [22, 23]. The number and maximum short-axis diameter for each station as well as location (upper zones, lower zones, or both) of LNE were recorded. When available, follow up CTs were also reviewed for the presence of LNE to evaluate the longitudinal behaviour of LNE. Radiologic patterns were also assessed according to the current ATS/ERS/ALAT/ JRS guidelines for diagnosis of IPF (UIP, probable UIP, Indeterminate for UIP and alternative diagnosis) [1]. All the evaluations were performed via consensus between two thoracic radiologists with expertise in ILD, blinded to clinical information of patients included in the study.

\section{Statistical analysis}

Assuming a survival rate of $70 \%$ in the group without mediastinal LNE over a follow up period of 2 years, a sample of 148 patients was calculated as required to provide the study with $90 \%$ power to detect a 2.26 -fold increased mortality risk in the group with mediastinal LNE with a two-sided significance level of $5 \%$. The effect size was chosen based on a previous study investigating the impact of mediastinal LNE on survival in IPF [22].

Means with standard deviations and frequencies or percentages were used as descriptives for continuous and categorical variables, respectively. T-test for continuous variables and the chi-square test for categorical variables were used to perform between-group comparisons of demographics and clinical data. Kaplan-Meier curves and the log-rank test were used to analyse the rates of mortality from any cause during the follow up and the rates of disease progression as defined by a categorical FVC decline $\geq 10 \%$ measured at the time of the last available pulmonary function assessment.

Crude and adjusted hazard ratios and their $95 \%$ confidence intervals (CIs) were calculated using Cox proportional hazard regression. Observed baseline and 12( \pm 4$)$ months FVC and percent predicted DLco values were entered in a mixed linear model with fixed effects for presence of mediastinal LNE, time, gender, age and random effects for patient-specific intercept to predict the annualized rate of change in the parameters for each patient. The effect of mediastinal LNE on FVC and DLco changes over time was determined on the basis of the time-by-group interaction term from the mixed model. $P$-values less than 0.05 were considered statistically significant. All analyses were performed using SPSS (version 24, IBM, USA).

\section{Results}

\section{Characteristics of study population}

One hundred eighty-one IPF patients from the ILD clinic registry were screened for eligibility (Fig. 1). Overall, twenty-nine patients were excluded for unavailability of CT scan within 12 months from diagnosis $(n=21)$, history of thoracic malignancy $(n=3)$, respiratory infection at the time of diagnosis $(n=1)$ or for missing reports of baseline pulmonary function tests $(n=4)$. One hundred fifty-two IPF patients in the ILD registry formed the final study population. The average clinical follow up time was 19.08 months (SD 12.8). Ninety-four (62\%) patients had at least one LNE on CT performed within a year from diagnosis, while 58 patients (38\%) had no LNE present. Within patient with mediastinal LNE, 62 (66\%) had two or more mediastinal lymph node with short-axis diameter $\geq 10 \mathrm{~mm}$, while 31 (33\%) had 3 or more LNE. In this group, the mean short-axis diameter of largest lymph node was $12.4 \mathrm{~mm}$ (SD 2.4). LNE was predominantly distributed in paratracheal stations ( $n=93,99 \%$ of LNE patients), while only 3 patients (3\%) had LNE in the lower zones. The large majority of patients included in this study $(n=135,89 \%)$ received antifibrotic treatment for IPF during the follow up.

Baseline characteristics of the study groups by presence of LNE on baseline CT scan are reported in Table 1. The two study groups did not present significant differences as to demographics. Patients without LNE had lower GAP stage $(p=0.038)$ and higher DLco \% predicted at baseline $(p=0.023)$ as compared to LNE+ patients. Diabetes was a more frequent comorbidity among patients with LNE $(25.8 \%$ vs $6.9 \%, p=0.004)$.

\section{All-cause mortality}

IPF patients with evidence of mediastinal LNE on baseline CT scan of the chest had lower survival rates as compared to patients without LNE (median survival 37.8 months vs 44.5 months, log-rank $p=0.025$ ) (Fig. 2). This corresponded to a 2.65-fold increased risk of mortality for LNE+ patients on Cox proportional hazard analysis (95\% CI 1.09-6.46, $p=0.032$ ) (Table 2). After stratification of the study population by number of enlarged mediastinal lymph nodes, the involvement of three or more lymph nodes was found significantly associated with worse survival as compared to each of the 


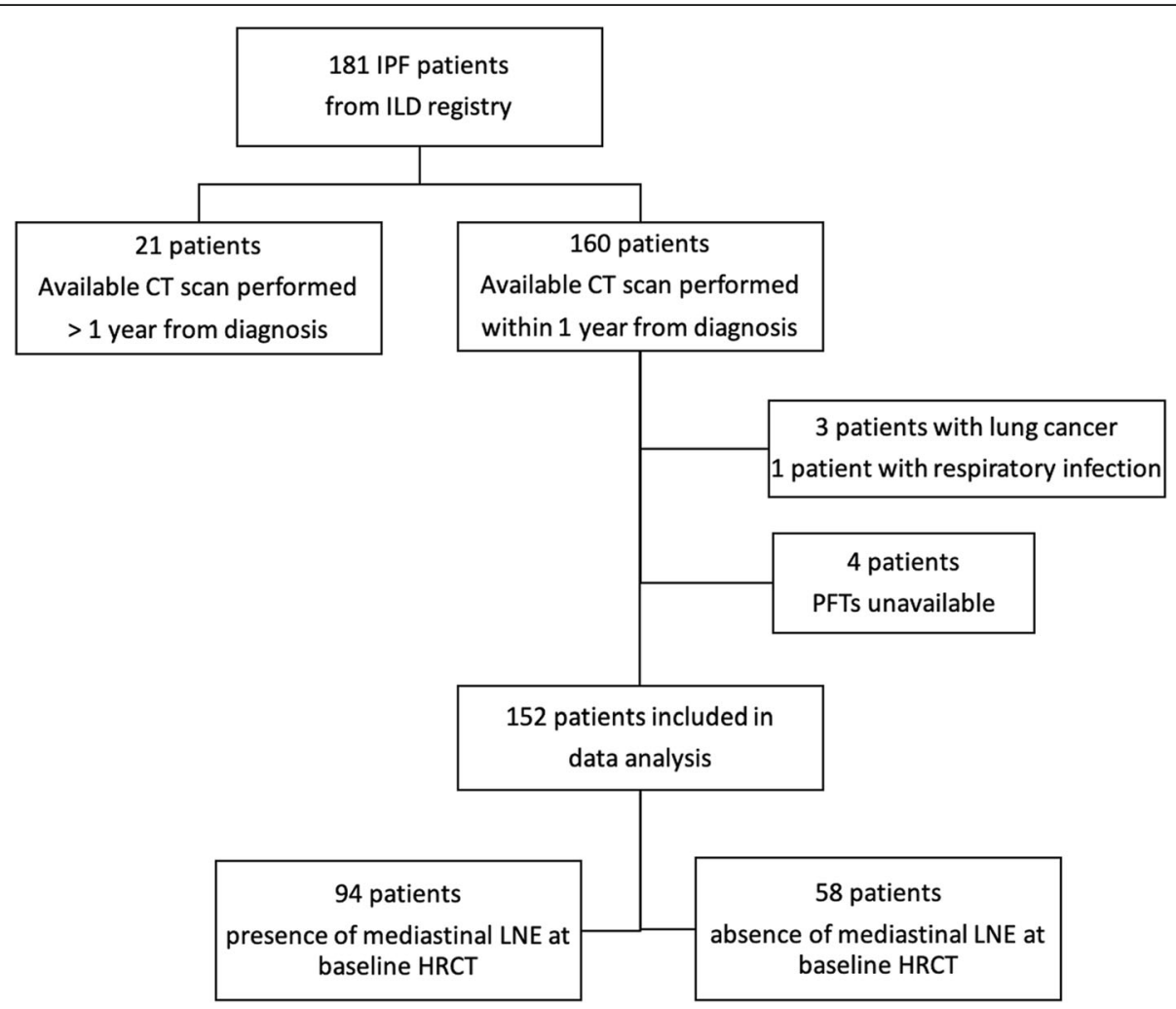

Fig. 1 Flowchart of patient selection for the study

other groups (Fig. 2), with a 5.72-fold increased risk of mortality (HR 5.72, 95\%CI 2.18-14.98, $p<0.001$ ). Within patients with mediastinal LNE at baseline, increasing dimensions of the largest lymph node were also associated with higher mortality (HR 1.17, 95\% CI 1.04$1.32, p=0.01$ ).

When hazard ratios were adjusted for GAP stage [8], only the presence of 3 or more mediastinal enlarged lymph nodes maintained a strong association with poorer survival (HR 5.03, 95\% CI 1.86-13.62, $p \leq 0.001$ ) (Table 2).

\section{Pulmonary function}

Patients with or without LNE presented similar functional decline as defined as $10 \%$ absolute decline in FVC (Fig. 3, Table 2). On the other hand, patients with evidence of 3 or more mediastinal enlarged lymph nodes showed increased disease progression rates both on univariate analysis (HR 2.67, 95\% CI 1.19-5.97, $p=0.017$ ) and after adjusting for GAP stage (HR 2.99, 95\% CI 1.22-7.33, $p=0.17$ ) (Table 2).

In order to further explore the association between mediastinal LNE and change in pulmonary function parameters, the annualized rates of change in absolute FVC and \% predicted DLco were compared between patients with different degrees of lymph node involvement at baseline (Fig. 4, Table 3). The rate of decline in FVC increased with the number of mediastinal lymph nodes involved and was largest in patients with 3 or more enlarged lymph nodes $(-178 \mathrm{~mL}$, SE 0.09), especially if compared to patients without LNE, who showed relative stability over 12 months (FVC change $-4 \mathrm{~mL}, \mathrm{SE}$ 0.07). However, the differences in the rates of change in FVC were not statistically significant as shown by the group-by-time interaction term in the model $(p=0.332)$. The largest decline in DLco occurred in the group with 3 or more enlarged lymph nodes $(-10.5 \%$, SE 2.55$)$ as compared with the other groups, although such difference did not meet statistical significance (group-by-time interaction $p=0.349$ ) (Table 3).

\section{Longitudinal assessment of mediastinal LNE}

One hundred seventeen patient cases who had available longitudinal imaging data were assessed for the presence of mediastinal LNE at the follow up CT scan. Average time difference between consecutive CT scans was 17.7 months (SD 10.4). LNE status did not change over time in $101(86 \%)$ of these patients. Among the 48 patients without evidence of mediastinal LNE at baseline, 38 (79\%) patients remained without mediastinal LNE at 
Table 1 Baseline characteristics of patients without (LNE-) or with (LNE+) mediastinal lymph node enlargement on CT scan of the chest. Data are expressed as counts (\%) or mean with standard deviation. Reported p-values were obtained via t-test for independent samples or Pearsons' Chi-squared test as applicable. BMI = body mass index; FVC = forced vital capacity; DLCO = diffusion lung capacity for carbon monoxide; $6 \mathrm{MWD}=6$-min walk distance; COPD = chronic obstructive pulmonary disease; OSAS = obstructive sleep apnea syndrome; GERD = gastroesophageal reflux disease; GAP = gender age physiology.

\begin{tabular}{|c|c|c|c|c|}
\hline & $\begin{array}{l}\mathrm{N} \text { available } \\
\text { observations }\end{array}$ & $\begin{array}{l}\text { LNE- } \\
(n=58)\end{array}$ & $\begin{array}{l}\text { LNE+ } \\
(n=94)\end{array}$ & $p$ value \\
\hline Age, years & 152 & $76(7.3)$ & $74.7(7.6)$ & 0.277 \\
\hline Sex & & & & 0.627 \\
\hline Male & 152 & $45(77.6)$ & $76(80.9)$ & \\
\hline Female & & $13(22.4)$ & $18(19.1)$ & \\
\hline Smoking history & & & & 0.599 \\
\hline Current & & $0(0)$ & $1(1.1)$ & \\
\hline Former & 148 & $34(60.7)$ & $60(65.2)$ & \\
\hline Never smoker & & $22(39.3)$ & $31(33.7)$ & \\
\hline BMI & 140 & $28.2(3.9)$ & $27.31(4.5)$ & 0.244 \\
\hline Use of oxygen therapy & & & & 0.405 \\
\hline No & 137 & $51(91.1)$ & $70(86.4)$ & \\
\hline Yes & & $5(8.9)$ & 11(13.6) & \\
\hline HRCT pattern & & & & 0.504 \\
\hline UIP & 136 & $27(52.9)$ & $43(50.6)$ & \\
\hline Probable UIP & & $14(27.5)$ & $32(37.6)$ & \\
\hline Indeterminate for UIP & & $7(13.7)$ & $7(8.2)$ & \\
\hline Alternative diagnosis & & $3(5.9)$ & $3(3.5)$ & \\
\hline Anti-fibrotic treatment & & & & 0.064 \\
\hline No treatment & 150 & $6(10.5)$ & $9(9.7)$ & \\
\hline Pirfenidone & & $30(52.6)$ & $32(34.4)$ & \\
\hline Nintedanib & & $21(36.8)$ & $52(55.9)$ & \\
\hline Follow up time (months) & 140 & $21.2(13.4)$ & $18.1(12.4)$ & 0.166 \\
\hline FVC volume, $L$ & 141 & $2.6(0.7)$ & $2.56(0.73)$ & 0.737 \\
\hline FVC \% predicted & 152 & $85.6(21)$ & $80.3(19.5)$ & 0.118 \\
\hline DLco \% pred & 142 & $58.9(23.2)$ & $50.1(21.7)$ & 0.023 \\
\hline $\mathrm{SaO}_{2} \%$ at rest & 142 & $94.4(2.2)$ & $94.7(2.7)$ & 0.563 \\
\hline \multicolumn{5}{|l|}{ Comorbidities } \\
\hline COPD & 152 & $4(6.9)$ & $5(5.3)$ & 0.732 \\
\hline Emphysema & 152 & $5(8.6)$ & $13(13.8)$ & 0.334 \\
\hline OSAS & 152 & $6(10.3)$ & $8(8.5)$ & 0.704 \\
\hline Chronic heart disease & 152 & $17(29.3 \%)$ & $32(34)$ & 0.544 \\
\hline Pulmonary hypertension & 152 & $2(3.4)$ & $12(12.8)$ & 0.054 \\
\hline GERD & 152 & $16(27.6)$ & $30(31.9)$ & 0.573 \\
\hline Anxiety/Depression & 152 & $2(3.4)$ & $4(4.3)$ & 0.804 \\
\hline Diabetes & 205 & $4(6.9)$ & $24(25.8)$ & 0.004 \\
\hline GAP Index & & & & 0.038 \\
\hline Stage 1 & 148 & $25(43.9)$ & $35(38.5)$ & \\
\hline Stage II & & $30(52.6)$ & $40(44.4)$ & \\
\hline Stage III & & $2(3.5)$ & $16(17.6)$ & \\
\hline
\end{tabular}

LNE number 
Table 1 Baseline characteristics of patients without (LNE-) or with (LNE+) mediastinal lymph node enlargement on CT scan of the chest. Data are expressed as counts (\%) or mean with standard deviation. Reported p-values were obtained via t-test for independent samples or Pearsons' Chi-squared test as applicable. BMI = body mass index; FVC = forced vital capacity; DLco = diffusion lung capacity for carbon monoxide; $6 \mathrm{MWD}=6$-min walk distance; $\mathrm{COPD}=$ chronic obstructive pulmonary disease; OSAS = obstructive sleep apnea syndrome; GERD = gastroesophageal reflux disease; GAP = gender age physiology. (Continued)

\begin{tabular}{llll}
\hline & $\begin{array}{l}\text { N available } \\
\text { observations }\end{array}$ & $\begin{array}{l}\text { LNE- } \\
(\boldsymbol{n}=\mathbf{5 8})\end{array}$ & $\begin{array}{l}\text { LNE+ } \\
(\boldsymbol{n}=\mathbf{9 4})\end{array}$ \\
\hline 1 & & & $32(34)$ \\
2 & & $31(33)$ \\
$\geq 3$ & & $-31(33)$ & $12.4(2.4)$ \\
Larger LNE $(\mathrm{mm})$ & 113 & - & 30.001 \\
\hline
\end{tabular}

follow up, while among the 69 patients with baseline mediastinal LNE 63 (91\%) maintained evidence mediastinal LNE over time. Among the 62 patients with baseline LNE who received antifibrotic treatment, 57 (92\%) had mediastinal LNE at follow up. Mediastinal LNE disappeared in only $6(9 \%)$ patients who had LNE at baseline, while $10(11 \%)$ patients who did not have LNE at baseline developed LNE at follow up.

\section{Discussion}

In this study we retrospectively investigated the impact of mediastinal LNE on survival and disease behaviour in patients with IPF followed up at a tertiary ILD centre in Italy. Our findings suggest that a diffuse involvement of mediastinal lymph nodes in IPF patients is an independent predictor of both mortality and functional deterioration in these patients.
Predicting the course of IPF represents a historical challenge for clinicians. Current risk prediction models, robustly developed using data from large randomized clinical trials, may not be suitable for individual prognostication in the real life heterogeneous IPF populations. Furthermore, no parameters have shown to be useful in predicting future trends of functional decline, the hallmark of progressive disease in IPF [13]. Mediastinal lymph node enlargement on CT scan has been recently investigated as a prognostic biomarker in IPF and other fibrotic ILD, for reflecting pathobiological mechanisms of activated immune response that may be relevant to easily stratify patient with at risk of poorer outcome. Sin and collagues [22] demonstrated that mediastinal LNE was a strong, independent predictor of mortality in a retrospective cohort of 132 patients with IPF. Adegunsoye and colleagues [23] nicely reported the association of several features of mediastinal lymphadenopathies with disease

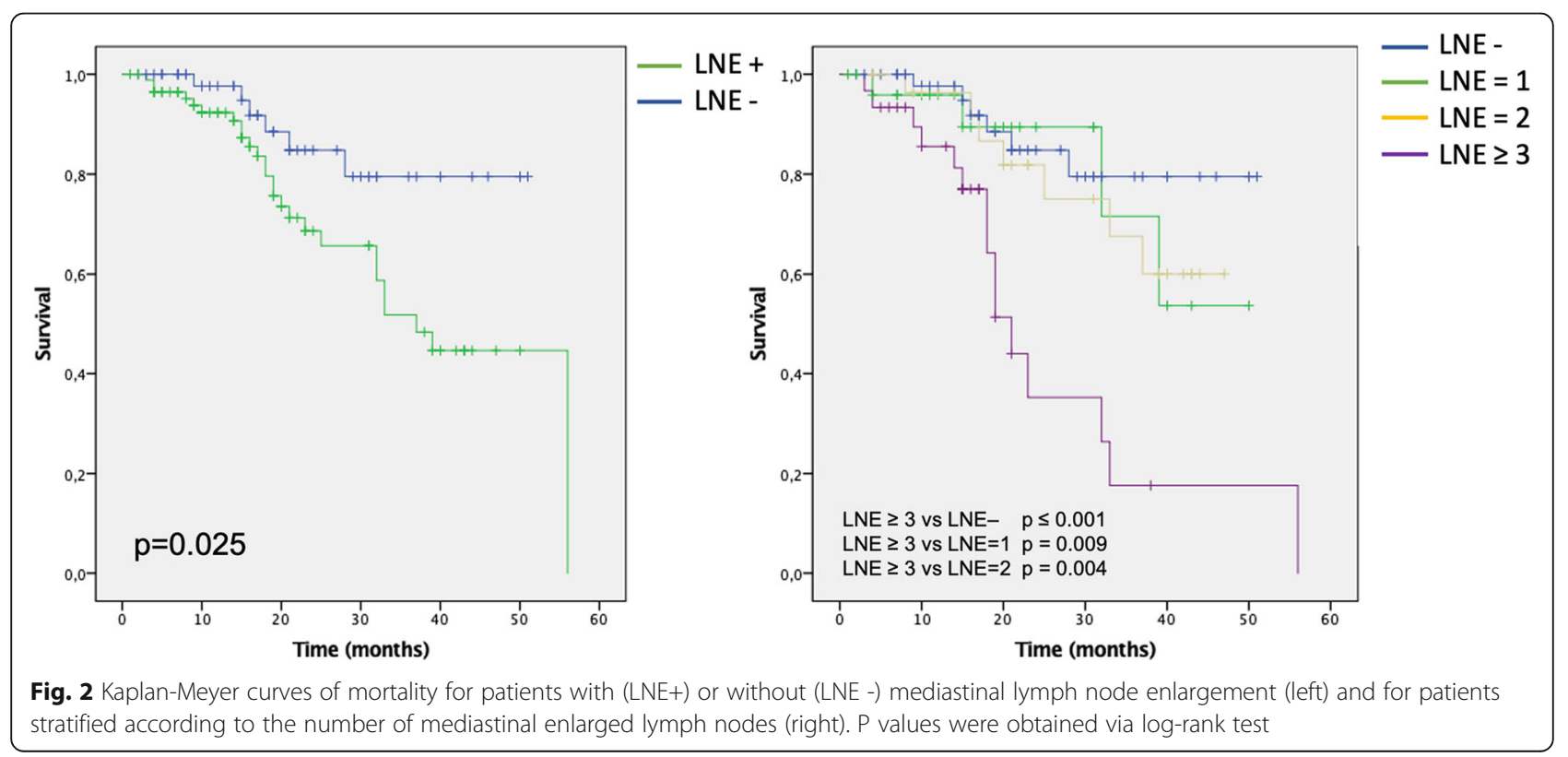


Table 2 - Cox proportional hazard regression analysis for all-cause mortality and disease progression. Disease progression was defined as death or absolute forced vital capacity (FVC) decline $\geq 10 \%$. *Values of HR were adjusted for GAP (Gender, Age, Physiology) stage index. ${ }^{* *}$ within LNE+ patients

\begin{tabular}{|c|c|c|c|c|c|c|c|c|}
\hline & \multicolumn{4}{|l|}{ Mortality } & \multicolumn{4}{|c|}{ Disease progression } \\
\hline & \multicolumn{2}{|l|}{ Unadjusted } & \multicolumn{2}{|l|}{ Adjusted* } & \multicolumn{2}{|l|}{ Unadjusted } & \multicolumn{2}{|l|}{ Adjusted* } \\
\hline & $\mathrm{HR}(95 \% \mathrm{Cl})$ & $p$ value & HR (95\% Cl) & $p$ value & HR $(95 \% \mathrm{Cl})$ & $p$ value & HR $(95 \% \mathrm{Cl})$ & $p$ value \\
\hline LNE + (vs LNE -) & $2.65(1.09-6.46)$ & 0.032 & $2.39(0.96-5.95)$ & 0.062 & $1.75(0.9-3.39)$ & 0.097 & $1.87(0.93-3.76)$ & 0.078 \\
\hline \multicolumn{9}{|l|}{ Number of LNE (vs 0) } \\
\hline 1 & $1.49(0.42-5.3)$ & 0.538 & $1.1(0.27-4.5)$ & 0.894 & $1.43(0.54-3.83)$ & 0.472 & $1.65(0.61-4.47)$ & 0.326 \\
\hline 2 & $1.62(0.54-4.84)$ & 0.386 & $1.55(0.52-4.66)$ & 0.436 & $1.41(0.63-3.17)$ & 0.405 & $1.53(0.67-3.52)$ & 0.313 \\
\hline$\geq 3$ & $5.72(2.18-14.98)$ & $<0.001$ & $5.03(1.86-13.62)$ & 0.001 & $2.67(1.19-5.97)$ & 0.017 & $2.99(1.22-7.33)$ & 0.017 \\
\hline Larger $\mathrm{LNE}^{* *}(\mathrm{~mm})$ & $1.17(1.04-1.32)$ & 0.01 & $1.14(0.99-1.29)$ & 0.058 & $1.05(0.89-1.23)$ & 0.57 & $0.93(0.84-1.18)$ & 0.928 \\
\hline
\end{tabular}

severity and higher risk of mortality or hospitalization in patients with a variety of forms of ILD, including IPF, IPAF (interstitial pneumonia with autoimmune features), CTD-ILD (connective tissue disease-associated interstitial lung disease) and unclassifiable ILD, suggesting a role for mediastinal lymph node assessment in ILD prognostication beyond specific etiology.

In our cohort, a "diffuse" lymph node involvement - as defined by 3 or more enlarged mediastinal lymph nodes - was strongly associated with lower survival rates, in line with previous findings. Sin and coworkers [22] demonstrated the impact of mediastinal LNE on survival in an equally-sized IPF cohort with comparable size and similar proportion of patients showing lymph node enlargement on CT. Notably though, the large majority of patients in our study received antifibrotic treatment after diagnosis, suggesting that the presence of multiple mediastinal lymphadenopathies may predict poorer prognosis despite treatment.

Importantly, our study provided first evidence as to the relationships between mediastinal LNE and disease progression expressed by means of functional decline. The involvement of 3 or more lymph nodes involved was found to be associated with an increased risk of disease progression as defined by significant drop $(\geq 10 \%)$ in percent predicted FVC, suggesting that diffuse mediastinal LNE represent a marker of more aggressive disease behaviour in these patients. Patients with higher number of lymph nodal stations involved also showed decreased annual trends of pulmonary function and gas diffusion capacity, although the slopes of change were not found significantly different as compared to patients with no or

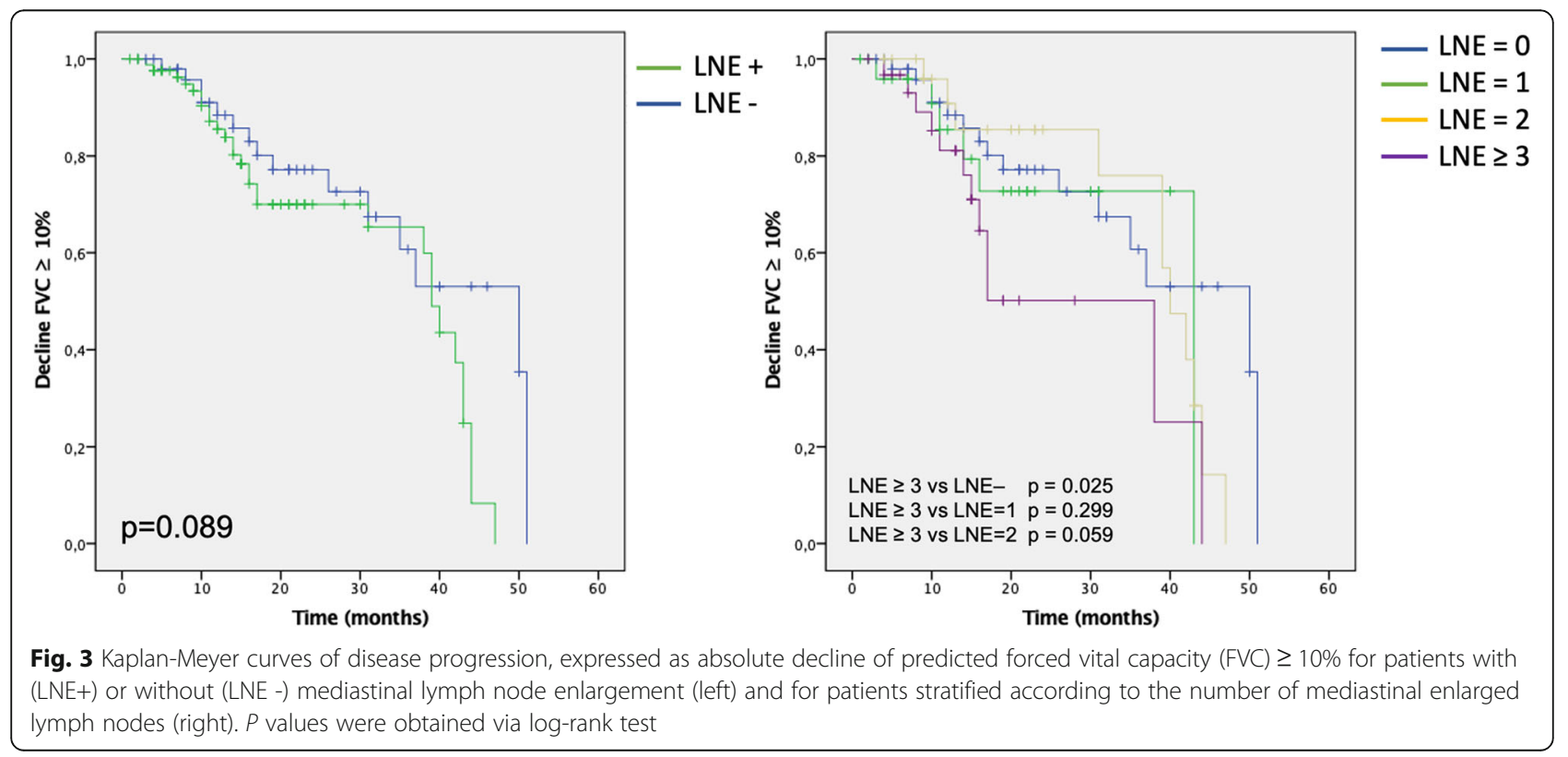




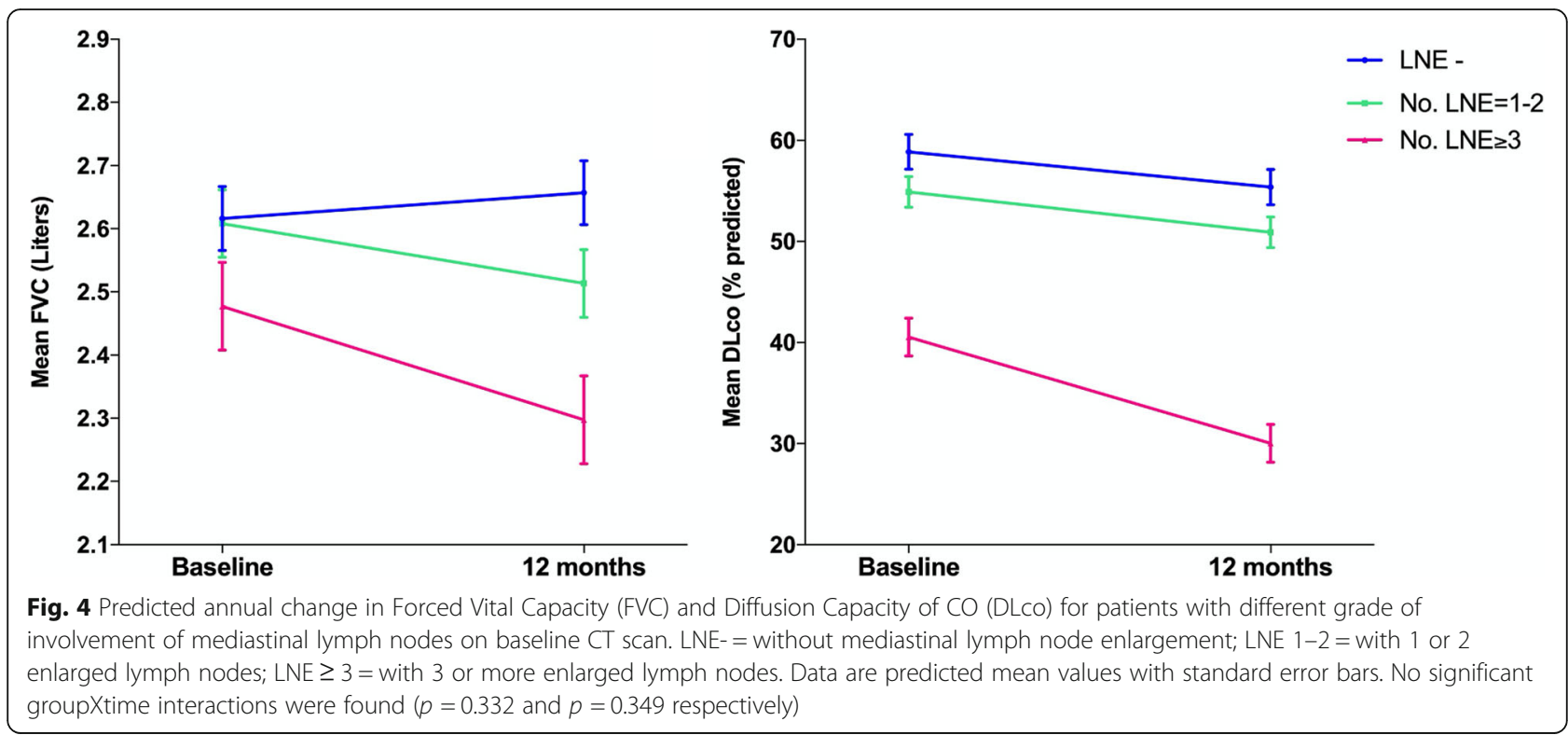

lesser lymph node involvement. Whilst our study was not powered to detect the impact of LNE on continuous change in pulmonary function parameters in a treated IPF population with reduced progression rates, these findings further support the hypothesis that major lymph node involvement predicts future functional deterioration.

The longitudinal evaluation of mediastinal LNE in a subpopulation with follow up imaging data showed that such finding is stable over time in most patients despite currently available antifibrotic treatment, which seems to preclude the role of LNE as a marker of response to treatment. Indeed, further studies are needed to clarify the pathobiological mechanisms at the basis of this prevalent finding in IPF patients. The study of histological features of LNE may help shed a light on the immune processes involved and whether a subset of patients exists where such pathways are paramount for driving disease progression.

The main limitations of our study are represented by its retrospective nature and the small size of our cohort.
Nevertheless, the study was adequately powered based on previously reports of mortality hazard ratio for IPF patients with LNE [22], which increases the robustness of our results. Another theoretical limitation consists in the heterogeneity of $\mathrm{CT}$ reconstruction protocols and contrast medium administration, as the exams have been performed in different centres.

\section{Conclusions}

We demonstrated that the presence of diffuse mediastinal lymphadenopathy on CT scan is independently associated with higher risks of mortality and clinically significant disease progression in a cohort of IPF patients despite antifibrotic treatment. Altogether, our results support the utility of mediastinal LNE in the risk stratification of the heterogeneous IPF population. Ongoing trials and future research will eventually clarify the pathobiology of mediastinal lymphadenopathy in IPF and if such finding may be useful to identify patients responding to treatments targeting specific immune pathways.

Table 3 Annualized rates of change in Forced Vital Capacity (liters) and DLco (\% predicted) across patients with different grade of involvement of mediastinal lymph nodes on baseline CT scan. LNE- = without mediastinal lymph node enlargement; LNE 1-2 = with 1 or 2 enlarged lymph nodes; LNE $\geq 3$ = with 3 or more enlarged lymph nodes. Data are means of predicted values (baseline-12 months) derived from linear mixed models. $p$ Values are for groupxtime interaction. $p$ Values $<0.05$ were considered statistically significant

\begin{tabular}{|c|c|c|c|c|}
\hline & \multicolumn{3}{|c|}{$\begin{array}{l}\text { 12-month } \\
\text { mean difference (SE) }\end{array}$} & \multirow[t]{2}{*}{$p$ value } \\
\hline & $\begin{array}{l}\text { LNE - } \\
n=58\end{array}$ & $\begin{array}{l}\text { LNE 1-2 } \\
n=63\end{array}$ & $\begin{array}{l}\mathrm{LNE} \geq 3 \\
n=31\end{array}$ & \\
\hline FVC (liters) & $-0.04(0.073)$ & $-0.095(0.073)$ & $-0.178(0.094)$ & 0.332 \\
\hline DLco (\% predicted) & $-3.49(2.35)$ & - $3.99(2.12)$ & $-10.5(2.55)$ & 0.349 \\
\hline
\end{tabular}




\section{Abbreviations}

LNE: Lymph Node Enlargement; IPF: Idiopathic Pulmonary Fibrosis; FVC: Forced Vital Capacity; HRCT: High-resolution computed tomography; ILD: Interstitial Lung Disease; PFTs: Pulmonary Function Tests; DLco: Diffusion capacity for carbon monoxide; GAP: Gender/Age/Physiology; IPAF: Interstitial pneumonia with autoimmune features; CTD-ILD: Connective tissue diseaseassociated interstitial lung disease

\section{Acknowledgments}

Not applicable.

\section{Authors' contributions}

Conceptualisation of the study: GS, ARL, FV, NG, MRC, LR. Data collection: NG, MRC. Analysis of radiological data: AF, ADC. Statistical Analysis: GS. Writing of study manuscript: GS. Review of study manuscript: BI, FV, ARL, RM, LR. Study supervision: LR, RM. All authors have read and approved the manuscript.

\section{Funding}

No external funding was provided for the conduction of this study.

\section{Availability of data and materials}

The datasets used and/or analysed during the current study are available from the corresponding author on reasonable request.

\section{Ethics approval and consent to participate}

The study was reviewed and received approval by the local ethics committee of Fondazione Policlinico Universitario "A. Gemelli" IRCCS in Rome. The need to receive an informed consent from all patients was waived due to the retrospective nature of the study.

\section{Consent for publication}

Not applicable.

\section{Competing interests}

Dr. Sgalla reports personal fees from Boheringer Ingelheim oustide the submitted work; Dr. Calvello reports personal fees from Boehringer Ingelheim, outside the submitted work; Dr. Varone reports personal fees from Boehringer Ingelheim, personal fees from Roche, outside the submitted work; Dr. lovene reports personal fees from Menarini, personal fees from Boehringer Ingelheim, outside the submitted work; Dr. Richeldi reports personal fees and other from Boehringer Ingelheim, grants and personal fees from InterMune, personal fees from Cipla, personal fees from Vertex, other from AstraZeneca, other from GlaxoSmithKline, other from Sanofi-Aventis, other from Celgene, other from Prometic, other from Roche, other from Takeda, during the conduct of the study. The rest of the authors declare no further competing interests.

\section{Author details}

'Dipartimento Scienze Gastroenterologiche, Endocrino-Metaboliche e Nefro-Urologiche, Unità Operativa Complessa di Pneumologia, Fondazione Policlinico Universitario "A. Gemelli" IRCCS, Rome, Italy. 'Dipartimento Diagnostica per Immagini, Radioterapia Oncologica ed Ematologia, Fondazione Policlinico Universitario A. Gemelli IRCCS, Rome, Italy. ${ }^{3}$ Dipartimento Universitario Scienze Radiologiche ed Ematologiche, Sezione di Radiologia, Università Cattolica del Sacro Cuore, Rome, Italy. ${ }^{4}$ Università Cattolica del Sacro Cuore, Rome, Italy.

Received: 21 July 2020 Accepted: 15 September 2020

\section{Published online: 21 September 2020}

\section{References}

1. Raghu G, Remy-Jardin M, Myers JL, Richeldi L, Ryerson CJ, Lederer DJ, et al. Diagnosis of idiopathic pulmonary fibrosis An Official ATS/ERS/JRS/ALAT Clinical practice guideline. Am J Respir Crit Care Med. 2018;198(5):e44.

2. Ley B, Collard HR, King TE. Clinical course and prediction of survival in idiopathic pulmonary fibrosis. Am J Respir Crit Care Med [Internet]. 2011; 183:431-40. Available from:. https://doi.org/10.1164/rccm.201006-0894Cl.

3. Raghu G, Collard HR, Egan JJ, Martinez FJ, Behr J, Brown KK, et al. An Official ATS/ERS/JRS/ALAT Statement: Idiopathic pulmonary fibrosis: Evidence-based guidelines for diagnosis and management. Am J Respir Crit Care Med
[Internet]. American Thoracic Society. 2011;183:788-824 [cited 2017 Jul 2] Available from: http://www.ncbi.nlm.nih.gov/pubmed/21471066.

4. King TE, Bradford WZ, Castro-Bernardini S, Fagan EA, Glaspole I, Glassberg MK, et al. A Phase 3 Trial of Pirfenidone in Patients with Idiopathic Pulmonary Fibrosis. N Engl J Med [Internet]. From the University of California, San Francisco, San Francisco (T.E.K.), InterMune, Brisbane (W.Z.B., E. A.F., E.G., D.K.), and Cedars-Sinai Medical Center, Los Angeles (P.W.N.) - all in California; Neumocare, Clinica San Borja, Lima, Peru (S.C.-B.); Alfr; 2014;370: 2083-92. Available from: https://doi.org/10.1056/NEJMoa1402582.

5. Richeldi L, du Bois RM, Raghu G, Azuma A, Brown KK, Costabel U, et al. Efficacy and Safety of Nintedanib in Idiopathic Pulmonary Fibrosis. N Engl J Med. 2014;370:2071-82. The authors' affiliations are listed in the Appendix Available from:. https://doi.org/10.1056/NEJMoa1402584.

6. Jegal Y, Dong SK, Tae SS, Lim CM, Sang DL, Koh Y, et al. Physiology is a stronger predictor of survival than pathology in fibrotic interstitial pneumonia. Am J Respir Crit Care Med. 2005;171:639-44.

7. Oda K, Ishimoto H, Yatera K, Naito K, Ogoshi T, Yamasaki K, et al. Highresolution $C T$ scoring system-based grading scale predicts the clinical outcomes in patients with idiopathic pulmonary fibrosis. Respir Res [Internet]. 2014;15:10 Available from: http://respiratory-research.com/ content/15/1/10

8. Ley B, Ryerson CJ, Vittinghoff E, Ryu JH, Tomassetti S, Lee JS, et al. A multidimensional index and staging system for idiopathic pulmonary fibrosis. Ann Intern Med. 2012;156:684-95 Department of Medicine, University of California, san Francisco, 505 Parnassus avenue, box 0111, San Francisco, CA 94143, USA. brett.ley@ucsf.edu Available from: http://www. ncbi.nlm.nih.gov/pubmed/22586007.

9. Jacob J, Bartholmai BJ, Rajagopalan S, van Moorsel CHM, van Es HW, van Beek FT, et al. Predicting Outcomes in Idiopathic Pulmonary Fibrosis Using Automated Computed Tomographic Analysis. Am J Respir Crit Care Med [Internet]. 1 Department of Respiratory Medicine. 2 Centre for Medical Image Computing, and. 3 Division of Radiology and. 4 St. Antonius ILD Center of Excellence, Department of Pulmonology, and. 5 Division of Heart and Lungs, University Medical Center Utrecht, Utrech: PG-0712-28073/ Department of Health/United Kingdom 20719/Arthritis Research UKUUnited Kingdom 2018/04/24 06:00 Am J Respir Crit Care Med. 2018 Sep 15;198(6): 767-776. doi: https://doi.org/10.1164/rccm.201711-21740C.; 2018;198:76776. Available from: https://www.ncbi.nlm.nih.gov/pubmed/29684284.

10. Ley B, Collard HR, King TE. Clinical course and prediction of survival in idiopathic pulmonary fibrosis. Am J Respir Crit Care Med. 2011.

11. Du Bois RM, Weycker D, Albera C, Bradford WZ, Costabel U, Kartashov A et al. Forced vital capacity in patients with idiopathic pulmonary fibrosis: test properties and minimal clinically important difference. Am J Respir Crit Care Med. 2011;184:1382-9.

12. Richeldi L, Ryerson CJ, Lee JS, Wolters PJ, Koth LL, Ley B, et al. Relative versus absolute change in forced vital capacity in idiopathic pulmonary fibrosis. Thorax. 2012:67:407-11.

13. Schmidt SL, Tayob N, Han MK, Zappala C, Kervitsky D, Murray S, et al. Predicting pulmonary fibrosis disease course from past trends in pulmonary function. Chest. 2014;145:579-85.

14. Salisbury ML, Xia M, Zhou Y, Murray S, Tayob N, Brown KK, et al. Idiopathic pulmonary fibrosis: Gender-age-physiology index stage for predicting future lung function decline. Chest [Internet]. Division of Pulmonary and Critical Care Medicine, Department of Medicine, University of Michigan, Ann Arbor, MI. Electronic address: msalisbu@med.umich.edu. Department of Biostatistics, University of Michigan, Ann Arbor, Ml. Department of Biostatistics, U; 2016;149:491-8. Available from: https://www.ncbi.nlm.nih. gov/pubmed/26425858.

15. Jacob J, Bartholmai BJ, Rajagopalan S, Kokosi M, Nair A, Karwoski R, et al. Automated Quantitative Computed Tomography Versus Visual Computed Tomography Scoring in Idiopathic Pulmonary Fibrosis. J Thorac Imaging [Internet]. 2016;31:304-11. Available from: http://content.wkhealth.com/ linkback/openurl?sid=WKPTLP:landingpage\&an=00005382-201609000-00005.

16. Jacob J, Bartholmai BJ, Rajagopalan S, Kokosi M, Nair A, Karwoski R, et al. Mortality prediction in idiopathic pulmonary fibrosis: evaluation of computer-based CT analysis with conventional severity measures. Eur Respir J [Internet]. 2017;49:1601011. Available from: http://www.ncbi.nlm.nih.gov/ pubmed/2781 1068\%5Cn http://erj.ersjournals.com/lookup/doi/10.1183/13 993003.01011-2016\%5Cn http://erj.ersjournals.com/lookup/doi/10.1183/13 993003.01011-2016. 
17. Jacob J, Bartholmai BJ, Rajagopalan S, van Moorsel CHM, van Es HW, van Beek FT, et al. Predicting outcomes in idiopathic pulmonary fibrosis using automated CT analysis Authors: Am J Respir Crit Care Med [Internet]. 2018, 1-56. Available from: http://www.atsjournals.org/doi/10.1164/rccm.201 711-21740C\%0A http://www.ncbi.nlm.nih.gov/pubmed/29684284.

18. Souza CA, Müller NL, Kyung SL, Johkoh T, Mitsuhiro H, Chong S. Idiopathic interstitial pneumonias: prevalence of mediastinal lymph node enlargement in 206 patients. Am J Roentgenol. 2006;186:995-9.

19. Lynch DA, Sverzellati N, Travis WD, Brown KK, Colby TV, Galvin JR, et al. Diagnostic criteria for idiopathic pulmonary fibrosis: a Fleischner society white paper. Lancet Respir Med. 2018.

20. Attili AK, Kazerooni EA, Gross BH, Flaherty KR, Martinez FJ. Thoracic lymph node enlargement in usual interstitial pneumonitis and nonspecificinterstitial pneumonitis: prevalence, correlation with disease activity and temporal evolution. J Thorac Imaging. 2006;21:288-92.

21. Bergin C, Castellino RA. Mediastinal lymph node enlargement of $C T$ scans in patients with usual interstitial pneumonitis. Am J Roentgenol. 1990.

22. Sin $\mathrm{S}$, Lee $\mathrm{KH}$, Hur JH, Hoon LS, Lee YJ, Jae CY, et al. Impact of mediastinal lymph node enlargement on the prognosis of idiopathic pulmonary fibrosis. PLoS One. 2018;13:e0201154.

23. Adegunsoye A, Oldham JM, Bonham C, Hrusch C, Nolan P, Klejch W, et al. Prognosticating Outcomes in Interstitial Lung Disease by Mediastinal Lymph Node Assessment: An Observational Cohort Study with Independent Validation. Am J Respir Crit Care Med [Internet]. University of Chicago, Section of Pulmonary and Critical Care, Dept. of Medicine, Chicago, Illinois, United States ; deji@uchicago.edu. University of California Davis Department of Internal Medicine, 158565, Pulmonary and Critical Care Medicine, Sacrament; 2018; Available from: https://www.ncbi.n/m.nih.gov/pubmed/3 0216085.

24. Walker CM, Chung JH, Abbott GF, Little BP, El-Sherief AH, Shepard JAO, et al. Mediastinal lymph node staging: From noninvasive to surgical. Am. J. Roentgenol. 2012:W54-64.

\section{Publisher's Note}

Springer Nature remains neutral with regard to jurisdictional claims in published maps and institutional affiliations.

Ready to submit your research? Choose BMC and benefit from:

- fast, convenient online submission

- thorough peer review by experienced researchers in your field

- rapid publication on acceptance

- support for research data, including large and complex data types

- gold Open Access which fosters wider collaboration and increased citations

- maximum visibility for your research: over $100 \mathrm{M}$ website views per year

At $\mathrm{BMC}$, research is always in progress.

Learn more biomedcentral.com/submissions 